\author{
G. Rotta ${ }^{1^{*}}$, T. Seramak ${ }^{2}$, K. Zasińska ${ }^{2}$ \\ ${ }^{1}$ Gdansk University of Technology, Faculty of Mechanical Engineering, Department of \\ Machine Design and Vehicles, 11/12 Narutowicza Street, 80-233 Gdansk, Poland \\ *grotta@pg.gda.pl \\ ${ }^{2}$ Gdansk University of Technology, Faculty of Mechanical Engineering, Department of \\ Materials and Welding Engineering, 11/12 Narutowicza Street, 80-233 Gdansk, Poland
}

\title{
ESTIMATION OF YOUNG'S MODULUS OF THE POROUS TITANIUM ALLOY WITH THE USE OF FEM PACKAGE
}

\begin{abstract}
Porous structures made of metal or biopolymers with a structure similar in shape and mechanical properties to human bone can easily be produced by stereolithographic techniques, e.g. selective laser melting (SLM). Numerical methods, like Finite Element Method (FEM) have great potential in testing new scaffold designs, according to their mechanical properties before manufacturing, i.e. strength or stiffness. An example of such designs are scaffolds used in biomedical applications, like in orthopedics' and mechanical properties of these structures should meet specific requirements. This paper shows how mechanical properties of proposed scaffolds can be estimated with regard to total porosity and pore shape.
\end{abstract}

Keywords: porous structures, Young’s modulus, Finite Element Method, titanium alloy

\section{INTRODUCTION}

Tissue engineering is a branch of biomedical science known for combining living tissue and engineered materials and one of its application is to use different types of scaffolds for reconstruction of bones. Using of porous structures are required for repairing the defective sites of the bones or for orthopaedic implants likes for Total Hip Replacements. Chosen scaffolds material must meet certain requirements like biocompatibility, features for cell proliferation, i.e. open porous structure for transportation of fluid, osteoconductive for growth of bone cells etc. In some cases biodegradability is required as well. Moreover, it should have sufficient strength and stiffness matched to the tissue cooperated with the scaffold. Materials for bone replacement should mimic the architecture of bone. A variety of biomaterials like metals (stainless steels, $\mathrm{Co}-\mathrm{Cr}$ alloys and titanium alloys), polymers, ceramics, and their combinations are successfully employed for fabrication of scaffolds.

However, the Young's modulus of biomaterials currently used is much larger than that of cortical bone, which causes stress shielding, bone atrophy and eventually implant loosening. Nowadays, orthopaedic implants are generally stiffer than the bone adjacent to the prosthesis. Due to its high stiffness, an implant prevents the applied stress from being evenly distributed 
over the contact area with the adjacent bone, thereby resulting in bone resorption around the implant. This weakness of implant support, leads to the bone fracture and implant loosening. Titanium and its alloys are attractive material for biomedical applications due to their excellent properties including high corrosion resistance, biocompatibility, osseointegration, excellent strength to weight ratio, good fracture toughness and high fatigue strength [12]. Moreover, titanium alloys have much lower values of Young's modulus and density than other metallic biomaterials.

Recently, many researchers have devoted notable efforts to prepare methodology of designing and manufacturing of porous structures that have mechanical properties similar to bone (especially stiffness and Young`s modulus). Porosity also provides proper environment for bone ingrowth in order to achieve a good fixation between porous implant and the surrounding bone. Nowadays, there are a number of manufacturing techniques for the fabrication of porous structures (both for metal and non-metal materials): selective laser melting (SLM) [8, 10, 14], selective laser sintering (SLS) [11], powder metallurgy (PM) [4], freeze casting [5], space holder [6], sponge replication [7]. These methods are applicable to other biomedical materials like stainless steels, biopolymers or ceramics as well [8, 10]. Combination of modern manufacturing techniques and Computer Aided Design (CAD) techniques makes possible to produce custom implant designs with individual, predesigned scaffolds and predefined macro- and microstructures for hard tissue engineering [10]. Porous titanium alloys are considered promising bone-mimicking biomaterials. Additive manufacturing techniques such as selective laser melting allow for manufacturing of porous titanium structures with a precise design of micro-architecture [15]. Example of porous scaffold made of titanium alloy Ti6Al4V and designed by Authors with the use of mentioned techniques is presented at Fig. 1.
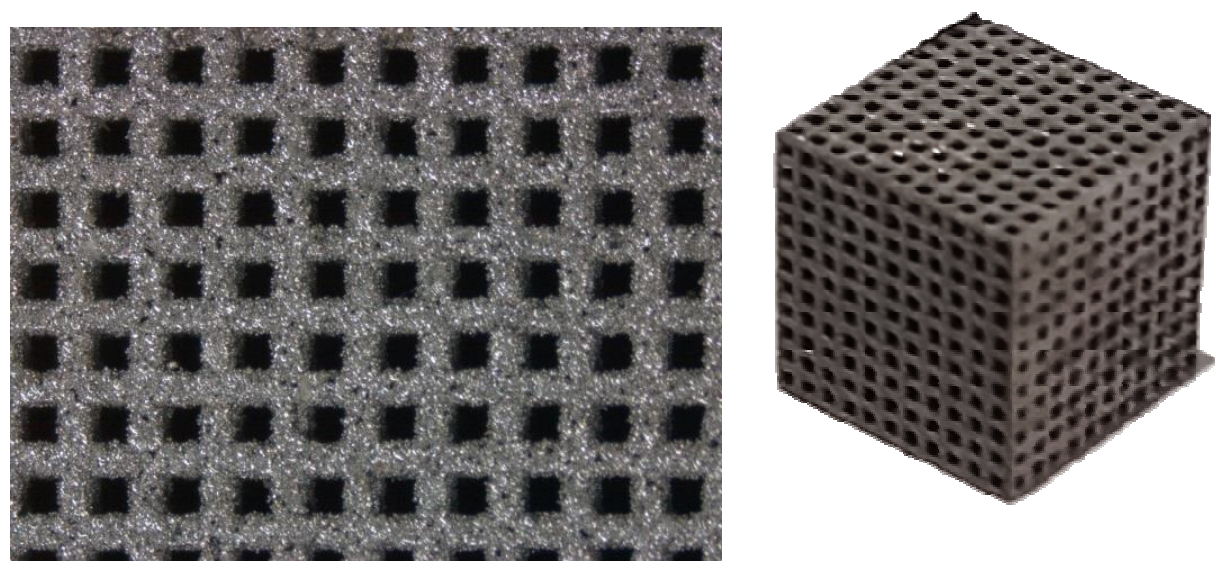

Fig. 1. Porous structure with cube pores made with the use of selective laser melting at the laboratory of Department of Material Engineering and Welding (Gdansk University of Technology)

Prediction of crucial mechanical parameters of designed scaffold is very important and relatively simple in case of regular porous structures built of sphere shaped or cube shaped primitives [13]. On the other hand it could be quite complicated for irregular scaffolds, when shapes are irregular or mixed or in case of intentionally varied porosity [1] like in cases of modelling of bone on the base of patient-specific 3D diagnostic images. 


\section{NUMERICAL ANALYSIS: GEOMETRICAL MODEL, MESH GRID AND BOUNDARY CONDITIONS}

Compression stress-strain test was computationally simulated in order to compare the mechanical properties of different types of porous scaffolds. Differences rely on the fact that scaffolds were designed from different types of primitives like spheres and cubes (Fig. 2). Authors decided to adopt a structure with interconnected, open pores of either spherical or cubic shapes. Sizes of these cavities, i.e. diameter or edge length were assumed as a pore size, whilst empty spherical or cubic spaces inside each elementary pore are "porosity makers" through variability of their dimensions.

a)

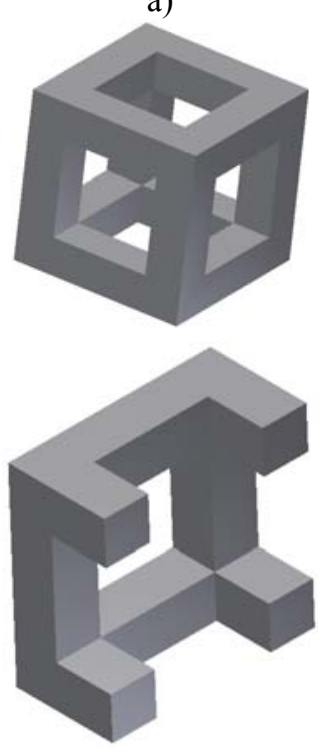

b)

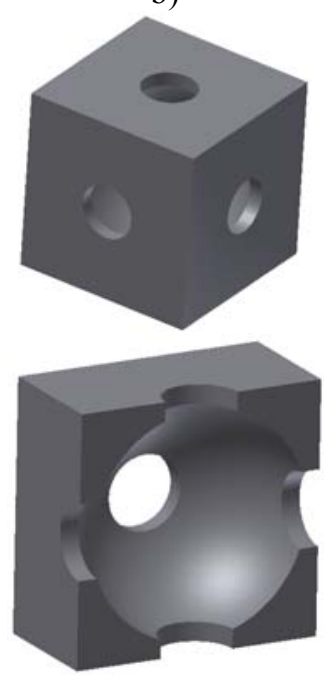

c)
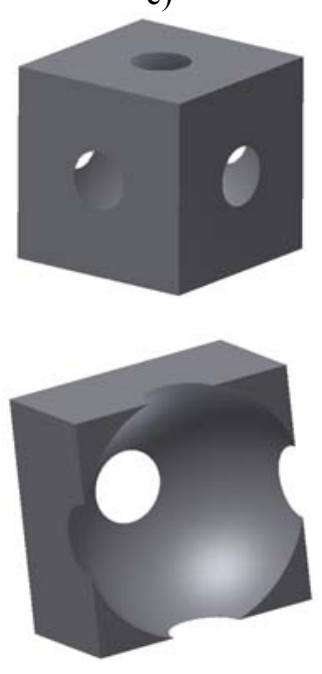

Fig. 2. Analyzed porous structures: a) cubic pores, b) spherical pores with interconnected channels - "heavy" type, c) spherical intersecting pores - "light" type

The size of a scaffold pore should be selected in such a range that growth of new bone cells within it could take place. The optimal value of pore size for bone ingrowth is $100 \div 600 \mu \mathrm{m}$ $[2,3,4,9,16]$. Scaffolds based on spherical pores were designed with assumption of some specific diameter $300 \mu \mathrm{m}$ of interconnected holes/channels. This is a significant limitation, thus the range of possible porosity is limited and different for both types of analyzed porous structures. Porosity of structure with channels between pores (named "heavy" spherical pore scaffold) could be varied in range of $18 \%-52 \%$, whilst intersecting pores (named "light" spherical pore scaffold) $52 \%-80 \%$. Increasing the dimensions of interconnected holes up to $500 \mu \mathrm{m}$, will extend the range of possible total porosity. Porosity $\mathrm{P}$ is calculated by the following formula Eq. 1:

where: $\mathrm{V}_{\text {void }}$ - is the volume of empty part of the pore, $\mathrm{V}_{\text {solid }}$ - is the volume of the metal part of the pore. 
Cubic pores had no limitation of $300 \mu \mathrm{m}$ size of interconnected holes, thus range of possible porosity was much wider. Comparison of pore sizes are shown on Fig. 3
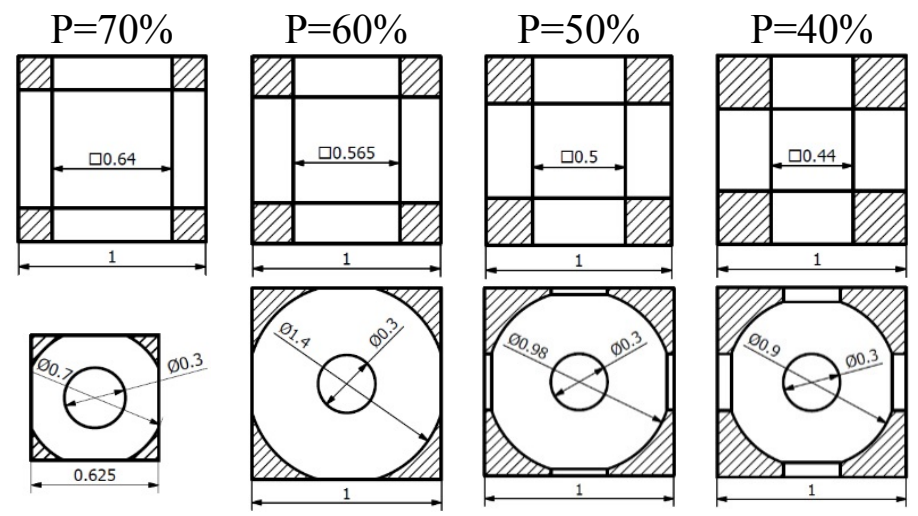

Fig. 3. Cross-sections through analyzed pores - comparison of profiles shapes and sizes

Finite element analysis software ANSYS 13.0 [ANSYS Inc. (2010)] was used to perform simulations on newly designed scaffold models to predict their mechanical properties. Threedimensional finite element models of regular structured cell units were used. The unit geometry used in simulations was $5 \times 5 \times 5 \mathrm{~mm}$ with different number of pores holes on each face, depended on types of pores and porosity of the structure. In fact, computed model is only one eight of the prototype 10x10x10 mm scaffold (Fig. 4). This is made possible by symmetric boundary condition, and, as a result, one can significantly save on computation time. The assumption of the ten millimeters characteristic dimension of scaffold sample, is due to prospective plans of comparison FEA computed results with experimental investigations of compression tests on scaffolds printed with the use of SLM technique, and $10 \mathrm{~mm}$ cube seems to be a suitable dimension with regard to feasibility of mechanical testing (especially thoroughness of deformation measurements) and the size of the numerical model.

Each rectangular shaped structure (Fig. 2a) and spherical pores with interconnected channels (Fig. 2b) had a $5 \times 5$ matrix of pores on each face (each elementary pore had dimensions of $1 \times 1 \times 1 \mathrm{~mm})$. On the other hand spherical intersected pores had varying number of pores on each face, i.e. for $60 \%$ porosity structure it was $5 \times 5$, whilst for $70 \%$ porosity it was $10 \times 10$, (that is each elementary pore had dimensions $0.5 \times 0.5 \times 0.5 \mathrm{~mm}-$ see Fig. 3). The scaffold models were generated in Autodesk Inventor 2012, and converted to SAT format and then imported to ANSYS for finite element analysis.

Solid tetrahedral elements SOLID185 were used in the analysis. This is a 4-node solid element (pyramid element), which provides three translational degrees of freedom for each node [Ansys Inc. (2010)]. Hexahedral elements (brick elements) are more accurate than pyramids, because they are less sensitive to the number of elements and category of elements (i.e. low- or high- order elements), more control on mesh quality (i.e. smaller amount of elements, shorter computational time) [13], but unfortunately CAD produced geometries, in many cases are not suitable for mapped meshing. The number of elements depends on the specific scaffold structure, but it is approximately equal to 1 million elements. Calculation time of each case was c.a. 5 minutes on computer with 2 DualCore AMD Opteron 275 processors and 12 GB RAM memory. After the geometrical model was 
imported, material properties, types and size of elements and boundary conditions were defined. Material data used in calculation are adequate to titanium alloy Ti6Al4V: Young`s modulus of base material $E=110 \mathrm{GPa}$, Poisson number $v=0.33$, density $\rho=4500 \mathrm{~kg} / \mathrm{m} 3$, loading force $\mathrm{F}=400 \mathrm{~N}$. As it was mentioned above, constraints were set as symmetric boundary conditions, whilst loading of the model was realized through forces imposed on nodes on the end face of the sample to simulate compression. Thus, von Misses stresses and displacements were the results of the calculation for each simulated sample. The relationship of the apparent elastic modulus $E^{*}$ of each porous specimen can be written as Eq. 2:

$$
\mathbf{E}^{*}=\frac{\mathbf{F}}{\Delta \mathbf{l}} \cdot \frac{\mathbf{L}}{\mathbf{A}}\lceil\mathrm{MPa}\rceil
$$

Here, $\mathrm{F}[\mathrm{N}]$ is the compressive force - input data, $\Delta \mathrm{l}[\mathrm{mm}]$ is the change in length of the specimen - result of calculation, $\mathrm{L}[\mathrm{mm}]$ is the length of the porous part of analyzed specimen $-5 \mathrm{~mm}$ in presented model, A $\left[\mathrm{mm}^{2}\right]$ is the total area (solid and holes) of the cross-section of the porous structure.

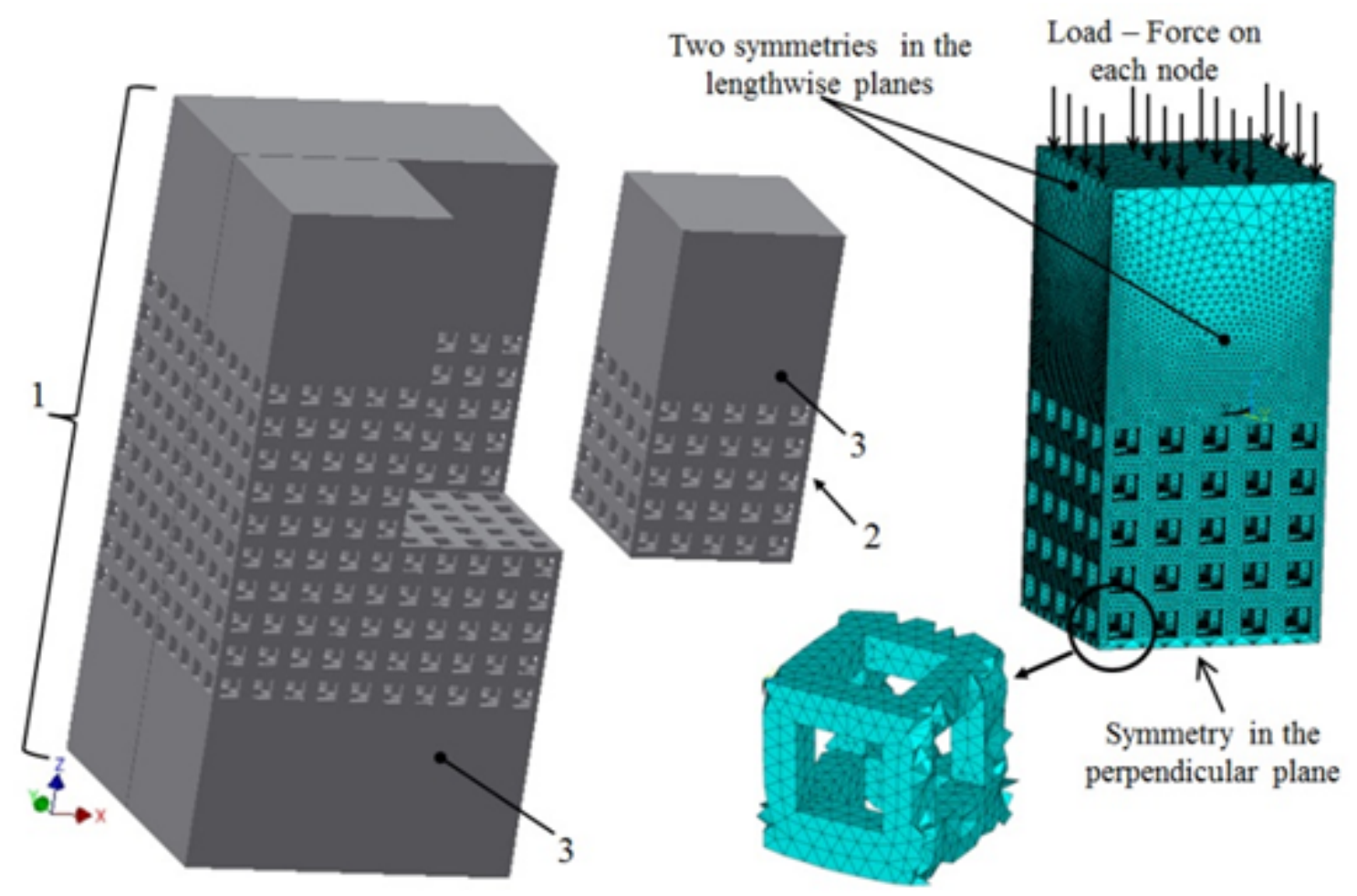

Fig. 4. Boundary conditions and details of element grid. Theoretically examined model

(1) $-10 \times 10 \times 10 \mathrm{~mm}$ scaffold; the section of the model actually analyzed (2) - it is possible due to symmetry during axial compression. The main purpose of the solid volumes (3) at both ends of the specimen is to shift the boundary conditions (represented by forces concentrated in element nodes) away from the actual porous structure 


\section{NUMERICAL ANALYSIS: RESULTS AND DISCUSSION}

Scaffolds' apparent Young`s modulus varies in range 17.1 $\div 49.3 \mathrm{GPa}$, where elastic modulus of base material was $110 \mathrm{GPa}$. It means, that under assumed conditions a reduction of Young`s modulus by $85 \%$ was achieved.

Table 1. Finite Element Analysis results

\begin{tabular}{|c|c|c|c|c|c|}
\hline & $\begin{array}{c}\text { Porosity } \\
{[\%]}\end{array}$ & $\begin{array}{c}\text { Apparent } \\
\text { density } \\
\rho^{*}\left[\mathrm{~kg} / \mathrm{m}^{3}\right]\end{array}$ & $\begin{array}{c}\text { Apparent } \\
\text { elastic } \\
\text { modulus E } \\
{[\mathrm{MPa}]}\end{array}$ & $\begin{array}{c}\text { Maximum } \\
\text { compressive } \\
\text { stress } \\
\sigma_{\max }[\mathrm{MPa}]\end{array}$ & $\begin{array}{c}\text { Axial strain } \\
\varepsilon[-] \\
\text { (relate to } 10 \mathrm{~mm} \\
\text { length scaffold } \\
\text { part) }\end{array}$ \\
\hline \multirow{3}{*}{$\begin{array}{c}\text { Cube-like } \\
\text { pores }\end{array}$} & 50 & 2700 & 45.2 & 18.7 & $4.43 \cdot 10^{-5}$ \\
\cline { 2 - 6 } & 60 & 1800 & 25.4 & 32.4 & $5.81 \cdot 10^{-5}$ \\
\cline { 2 - 6 } & 70 & 1350 & 17.1 & 39.8 & $7.87 \cdot 10^{-5}$ \\
\hline $\begin{array}{c}\text { Spherical } \\
\text { "heavy" } \\
\text { pores }\end{array}$ & 40 & 2700 & 49.3 & 26.2 & $4.06 \cdot 10^{-5}$ \\
\cline { 2 - 6 } & 50 & 2250 & 40.4 & 37.3 & $4.95 \cdot 10^{-5}$ \\
\hline $\begin{array}{c}\text { Spherical } \\
\text { "light" } \\
\text { pores }\end{array}$ & 60 & 1800 & 30.3 & 49.1 & $6.61 \cdot 10^{-5}$ \\
\cline { 2 - 6 } & 70 & 1350 & 21.1 & 56.3 & $9.46 \cdot 10^{-5}$ \\
\hline
\end{tabular}

Fig. 5a and Fig. 5b show contours of stresses in scaffold with cube-like pores; those contours are very similar in appearance shapes for all porosities of this scaffold type, only the values of stresses and displacements are altered by porosity change.

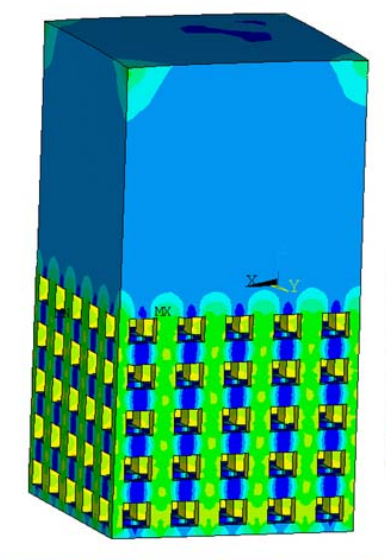

0.4 a)

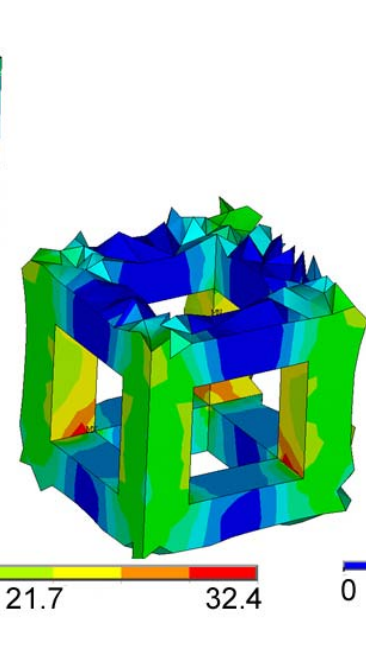

b)

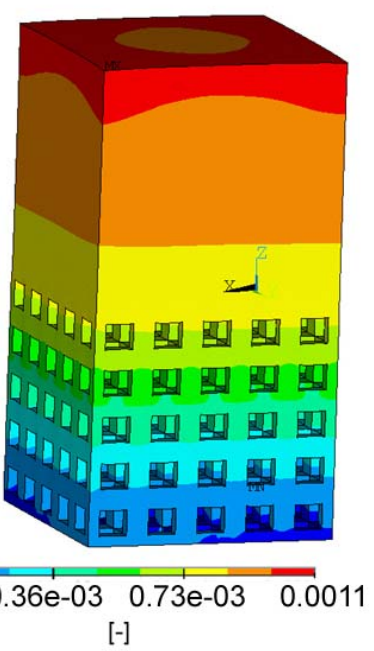

Fig. 5. Distribution of: a) Von Misses stress, b) displacement of the $60 \%$ porosity cube-like pores scaffold 
Fig. $6 \mathrm{a}$ and Fig. $6 \mathrm{~b}$ show contours of stresses in scaffold with spherical pores; likewise cubic pores, appearance of stress and displacement contours are very similar for all porosities of this scaffold type, but their values alter with porosity change. For adequate porosities higher values of maximum stress were noticed in cases of spherical pores especially for "light" spherical pores, and the difference could reach the level of $50 \%$. This effect is always visible at the edge of the interconnected window, where in fact in perfect model the edge is very sharp, thus the angles in corners of some elements could be very small, and, as a result, a zero curvature of radius between edges which meet at a corner, thus computed stress approaches relatively higher values than in finer meshed cubic pores. During manufacture of the actual scaffold it would be difficult to make the edges of the interconnected holes with designed thickness, because particles of melted powder size are between 30 and $50 \mu \mathrm{m}$, and in fact differences in stresses are much smaller. As it was showed in [13] strains are independent of the element shapes.

a)

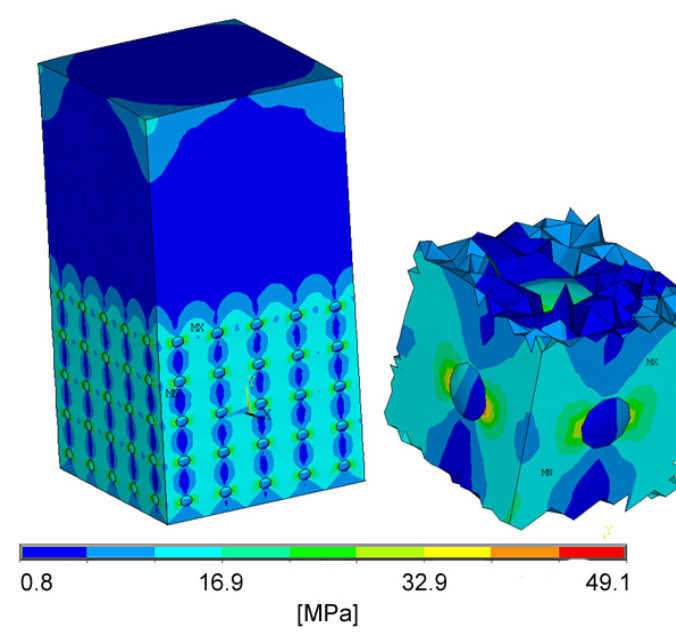

b)

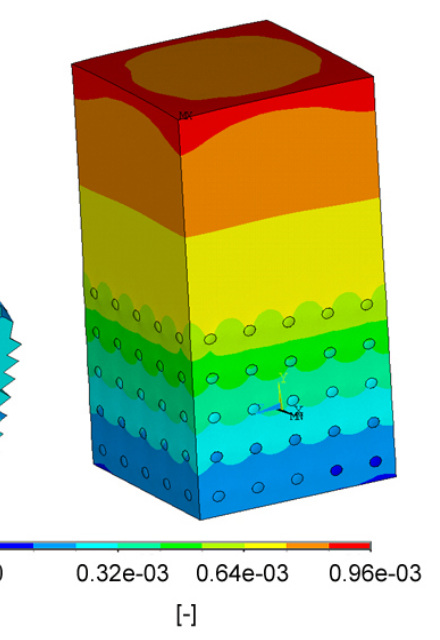

Fig. 6. Distribution of: a) Von Misses stress, b) displacement of spherical pores scaffold at $60 \%$ porosity

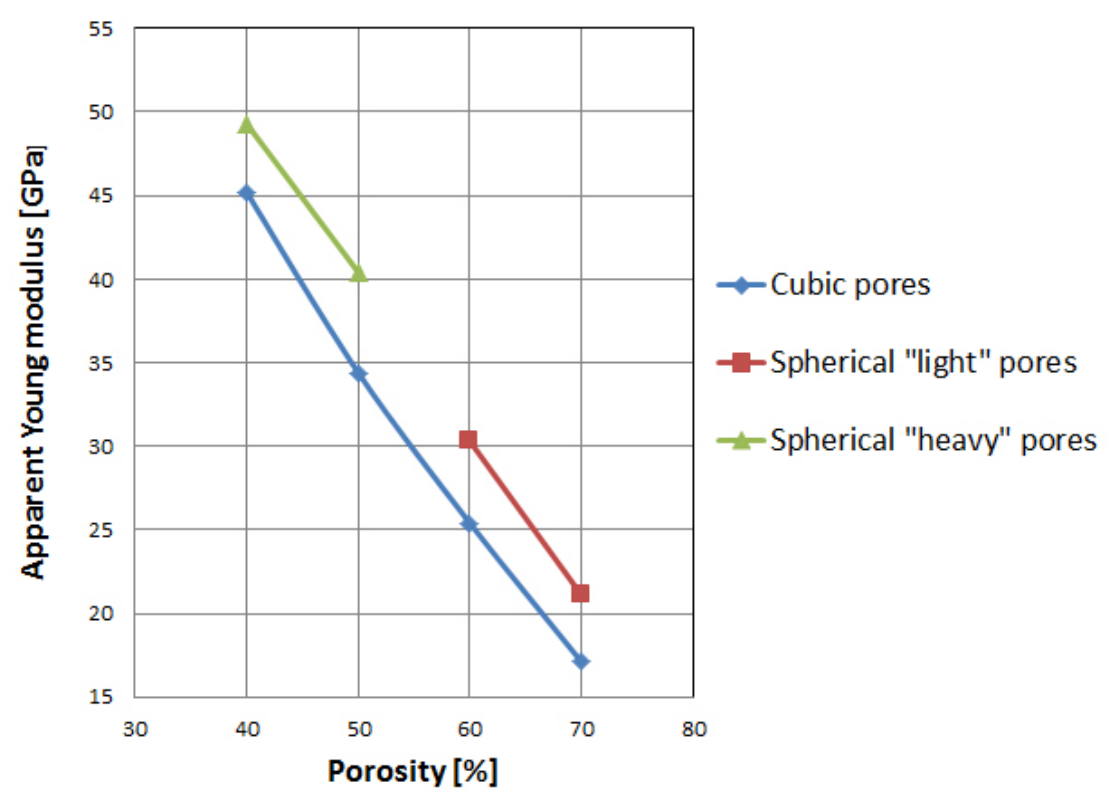

Fig. 7. Influence of the porosity on elastic modulus 
Fig. 7 shows relation of apparent Young`s modulus to total porosity. One can see that in analyzed range of porosity, which cover the range of medical applications, the variability of the apparent Young`s modulus is nearly linear, and the cubic and spherical curves are nearly parallel to each other and shifted by c.a. $10 \mathrm{GPa}$.

\section{SUMMARY}

A 3D numerical model of different types of regular scaffolds has been developed. Application of the model demonstrated strong influence of the pore size on the elastic modulus. Change of the Young`s modulus, in the studied range of porosity reached approximately $85 \%$ decrease in comparison to base Young`s modulus. In case of $70 \%$ porosity apparent Young s modulus achieved the value of $17.1 \mathrm{GPa}$. The shape of pores has less influence than the pore size, but so called "heavy" spherical pores gave c.a. $15 \%$ higher values of elasticity module than corresponding cube-like pores.

\section{REFERENCES}

1. Arabnejad Khanoki S., Pasini D.: Multiscale design and multiobjective optimization of orthopaedic hip implants with functionally graded cellular material. Journal of Biomechanical Engineering 134 (2012).

2. Bram M., Schiefer H., Bogdanski D. et al.: Implant surgery: How bone bonds to PM titanium. Metal Powder Report 61 (2006) 26-28, 30-31.

3. Biomet: Regenerex Brochure, Warsaw, 2008.

4. Dabrowski B., Swieszkowski W., Godlinski D., Kurzydlowski K. J.: Highly porous titanium scaffolds for orthopaedic applications. J. of Biomedical Materials Research Part B 95A (2012) 5361.

5. Deville S.: Freeze-Casting of Porous Biomaterials: Structure, Properties and Opportunities. Materials 3 (2010) 1913-1927.

6. Dezfuli S.N., Sadrnezhaad S.K. i in.: Fabrication of biocompatible titanium scaffolds using space holder technique. J Mater Sci Mater Med. 23 (2012) 2483-2488.

7. Li J.P., Wijn J.R., van Blitterswijk C. A., de Groot K.: Comparison of Porous Ti6Al4V Made by Sponge Replication and Directly 3D Fiber Deposition and Cancellous Bone. Key Engineering Materials 330-332 (2007) 999-1002.

8. Li R., Shi Y., Wang Z. i in.: Densification behaviour of gas and water atomized 316L stainless steel powder during selective laser melting. Applied Surface Science 256 (2010) 4350-4356.

9. Lin C.-Y., Wirtz T., LaMarca F., Hollister S.J: Structural and mechanical evaluations of a topology optimized titanium interbody fusion cage fabricated by selective laser melting process. Journal of Biomedical Materials Research Part A 83A (2007) 272-279.

10. Lindner M., Hoeges S, Meiners W. i in.: Manufacturing of individual biodegradable bone substitute implants using selective laser melting technique. Journal of Biomedical Materials Research Part A 97A (2011) 466-471. 
11. Liu F.-H., Lee R.-T., Lin W.-H, Liao Y.-S.: Selective laser sintering of bio-metal scaffold. Procedia CIRP 5 (2013) $83-87$.

12. Maya A.E., Grana D.R., Kokubu G.A. i in.: Zr-Ti-Nb porous alloys for biomedical application. Materials Science and Engineering C 32 (2012) 321-329.

13. Rotta G., Seramak T.: On the necessity of experimental verification of numerical results in biomedical applications. Proceedings of the XIX National Conference Applications of Mathematics and Biology and Medicine, Gdansk, Poland, 2013, pp. 78-83.

14. Warnke P. H., Douglas T, Wollny P i in.: Rapid Prototyping: Porous Titanium Alloy Scaffold Produced by Selective Laser Melting for Bone Tissue Engineering. Tissue Engineering 15 (2009) $115-124$

15. Yavari S.A., Wauthle R., Riemslag A.C. i in.: Fatigue behaviour of porous biomaterials manufactured using selective laser melting. Materials Science and Engineering C 33 (2013) 48494858.

16. Zimmer, Trabecular Metal Brochure, Carlsbad, 2012. 